University of Nebraska - Lincoln

DigitalCommons@University of Nebraska - Lincoln

8-20-2007

\title{
Novel method for mechanical characterization of polymeric nanofibers
}

\author{
Mohammad Naraghi \\ University of Illinois at Urbana Champaign, Urbana, Illinois \\ Loannis Chasiotis \\ University of Illinois at Urbana Champaign, Urbana, Illinois \\ Harold Kahn \\ Case Western Reserve University, Cleveland, Ohio \\ Yongkui Wen \\ University of Nebraska - Lincoln \\ Yuris A. Dzenis \\ University of Nebraska-Lincoln, ydzenis@unl.edu
}

Follow this and additional works at: https://digitalcommons.unl.edu/cmrafacpub

Part of the Nanoscience and Nanotechnology Commons

Naraghi, Mohammad; Chasiotis, Loannis; Kahn, Harold; Wen, Yongkui; and Dzenis, Yuris A., "Novel method for mechanical characterization of polymeric nanofibers" (2007). Faculty Publications from Nebraska Center for Materials and Nanoscience. 33.

https://digitalcommons.unl.edu/cmrafacpub/33

This Article is brought to you for free and open access by the Materials and Nanoscience, Nebraska Center for (NCMN) at DigitalCommons@University of Nebraska - Lincoln. It has been accepted for inclusion in Faculty Publications from Nebraska Center for Materials and Nanoscience by an authorized administrator of DigitalCommons@University of Nebraska - Lincoln. 


\title{
Novel method for mechanical characterization of polymeric nanofibers
}

\author{
Mohammad Naraghi and loannis Chasiotis \\ Aerospace Engineering, University of Illinois at Urbana Champaign, 325 Talbot Lab, 104 S. Wright Street, \\ Urbana, Illinois 61801 \\ Harold Kahn \\ Department of Materials Science and Engineering, Case Western Reserve University, Cleveland, \\ Ohio 44106-7204 \\ Yongkui Wen and Yuris Dzenis \\ Department of Engineering Mechanics, University of Nebraska, Lincoln, Nebraska 68588
}

(Received 24 April 2007; accepted 18 July 2007; published online 20 August 2007)

\begin{abstract}
A novel method to perform nanoscale mechanical characterization of highly deformable nanofibers has been developed. A microelectromechanical system (MEMS) test platform with an on-chip leaf-spring load cell that was tuned with the aid of a focused ion beam was built for fiber gripping and force measurement and it was actuated with an external piezoelectric transducer. Submicron scale tensile tests were performed in ambient conditions under an optical microscope. Engineering stresses and strains were obtained directly from images of the MEMS platform, by extracting the relative rigid body displacements of the device components by digital image correlation. The accuracy in determining displacements by this optical method was shown to be better than $50 \mathrm{~nm}$. In the application of this method, the mechanical behavior of electrospun polyacrylonitrite nanofibers with diameters ranging from 300 to $600 \mathrm{~nm}$ was investigated. The stress-strain curves demonstrated an apparent elastic-perfectly plastic behavior with elastic modulus of $7.6 \pm 1.5 \mathrm{GPa}$ and large irreversible strains that exceeded $220 \%$. The large fiber stretch ratios were the result of a cascade of periodic necks that formed during cold drawing of the nanofibers. (C) 2007 American Institute of Physics. [DOI: 10.1063/1.2771092]
\end{abstract}

\section{INTRODUCTION}

Polymeric nanofibers are an emerging class of building block materials with applications in tissue engineering, ${ }^{1,2}$, filtration, ${ }^{3}$ and nanocomposites. ${ }^{4}$ They are fabricated by drawing, producing relatively long individual nanofibers in a batch process, ${ }^{5}$ by template synthesis resulting in nanofibers that are tens of microns in length, ${ }^{6}$ and by electrospinning, ${ }^{7}$ allowing for mass production of long polymeric nanofibers in a continuous nonwoven form. In electrospinning, a polymeric melt or solution is ejected from a capillary toward a metal target by applying high voltage between the capillary and the target. As the jet travels to the target, it undergoes several instabilities that result in thin nanofibers with diameters ranging from a few tens to hundreds of nanometers after solidification. It is expected that the process parameters ${ }^{7,8}$ influence the elastic/plastic and time-dependent mechanical properties of the fibers and as a result their hierarchically structured macroscopic applications. During fabrication, the polymer jet undergoes high elongation rates and large reduction in cross section, which may result in molecular orientation along the fiber axis. ${ }^{9}$ These fabrication conditions coupled with the large surface-to-volume ratio of individual nanofibers prompt a spectrum of mechanical behaviors that can deviate significantly from bulk. The enhancement in axial fiber properties can be taken advantage of with the aid of fiber collectors designed to gather a spool of nanofibers. ${ }^{8,10}$

A variety of approaches has been applied towards me- chanical characterization of nanofibers and nanowires by employing nanoindentation, bending tests, resonance frequency measurements, and microscale tension tests. Nanoindentation has been used to measure the elastic moduli of onedimensional nanostructures such as $\mathrm{GaN}$ and $\mathrm{ZnO}$ nanowires and electrospun nanofibers. ${ }^{11,12}$ Although convenient, this method is not as accurate due to uncertainties stemming from the nanoindenter tip shape and the relative tip-fiber configuration, the effect of fiber surface curvature and roughness, and the adhesion force between the sample and the indenter. ${ }^{12}$ Furthermore, such local measurements do not provide input about the dominant mode of deformation and failure of nanofibers in their expected application which is axial stretching.

Three-point-bending ${ }^{12,13}$ and cantilever bending ${ }^{14}$ tests on nanofibers and nanowires have also been reported. These measurements may provide a mean to study the linearly elastic response of a fiber ${ }^{12,13}$ and its yield point. ${ }^{15}$ Although this method is a direct translation of macroscale testing of ceramic samples, the precise definition of boundary conditions at the nanofiber scale is unknown, and one can hardly speak about built-in supports that prevent fiber rotation or simply supported conditions that rule out fiber sliding.

While resonance frequency measurements of the material modulus have been successfully reported for metallic and ceramic wires, ${ }^{16,17}$ their application to polymeric fibers is not trivial because of the limited bending rigidity of the fibers that results in a whipping motion under lateral excita- 
tion. Recently, a novel method for measuring the linear response of single polymeric nanofibers has been reported, in which the shift in the resonance frequency of an attached atomic force microscope (AFM) cantilever was used to calculate the fiber stiffness. ${ }^{8}$

Finally, tension tests, following macroscale standards, involve the least number of assumptions necessary to extract material properties, and allow for fiber testing until failure. ${ }^{19}$ Due to the small nanofiber dimensions, several authors implemented a combination of AFM cantilevers as the load sensors ${ }^{16,20-22}$ to conduct tests inside a scanning electron microscope (SEM). ${ }^{22}$ Zussman et al. used this method to test carbon nanofibers from polymeric precursors and nanofibers ${ }^{20}$ under an optical microscope. ${ }^{16}$ Moreover, tension tests have been performed using a commercial apparatus. ${ }^{12,23,24}$ While convenient from an instrumentation viewpoint, the force range of commercial equipment can be prohibitive for nanofibers with diameters of a few hundreds of nanometers. ${ }^{12}$ Along the same lines, microelectromechanical system (MEMS) devices with on-chip force sensors and actuators have been used to perform tension tests of nanostructures: Lu et al. ${ }^{25}$ used a MEMS platform with a thermal actuator to test carbon nanotubes. The tests were performed inside an SEM for cross-head displacement measurement. Zhu et $\mathrm{al}^{26}$ developed a MEMS platform with a thermal actuator in which the applied force was measured by different capacitance method. Deformation of the samples was computed by comparing SEM images of the device recorded during loading. Kiuchi et al. developed a microdevice with an on-chip comb drive actuator for tension testing of nanowires. The authors used a lever mechanism to amplify the motion of the components of the device by a factor of 91 so that small deformations of the sample could be captured optically. ${ }^{27}$ The small form factor of on-chip actuators permits their use inside analytical chambers such as SEMs and TEMs, but the force and motion they can generate are limited, which makes them prohibitive for use with samples that allow for large stretch ratios. ${ }^{28,29}$ Recently, Samuel et al. reported on mechanical testing of pyrolyzed polymer nanofibers. ${ }^{30}$ They also utilized a microdevice with a leafspring load cell, which was actuated externally with a piezomotor. The average length of a sample was $10 \mu \mathrm{m}$, and the maximum engineering strain on the sample was $15 \%$. Because of the small sample length, an SEM was used for high magnification imaging to measure displacements. Their measurement was at the pixel level as opposed to the subpixel resolution of the method presented here. Furthermore, compared to the variable loading rate that can be accomplished by the approach presented in this article, the use of an SEM allows only for quasistatic tension tests as a result of its rastering action. In our work, the vulnerability of electrospun nanofibers to e-beam radiation, ${ }^{31}$ the large fiber ductility, ${ }^{20}$ and the strain rate dependent mechanical behavior of the electrospun polymers demanded testing outside an SEM. Thus, the method of subpixel displacement measurements applied here was required to conduct this category of experiments.

In the aforementioned microscale experimental methods, mechanical property measurements are often identified with

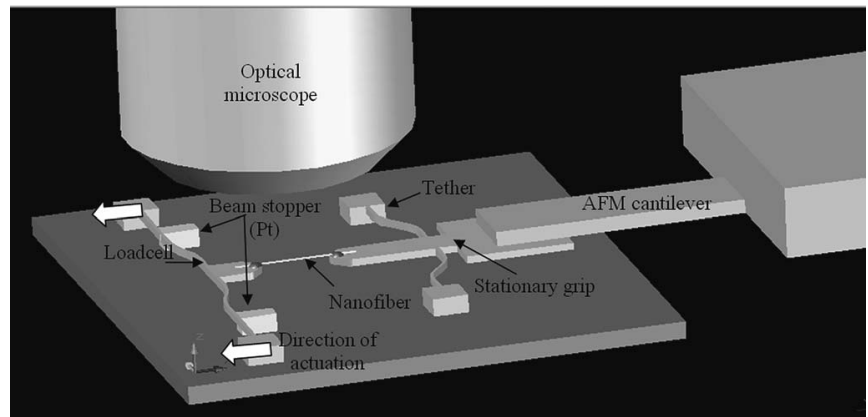

FIG. 1. Application of the test platform for nanofiber testing. The fiber length in this figure is $50 \mu \mathrm{m}$.

implementation inside an SEM which provides high magnification and, thus, resolution in displacements, but limits the capability for strain rate or time-dependent mechanical behavior studies due to the rastering function of an SEM. Conventional methodologies resort to pixel level measurements of load cell deflection and fiber length by using highresolution electron microscopy. Optical imaging, on the other hand, does provide rapid data recording, but the local displacement resolution is diffraction limited. However, even in the case of SEM imaging, it is the effective "cross-head" displacements that are recorded, which reduces the problem to that of monitoring the motion of the sample grips. To this effect, rigid body motions can be resolved from optical images with an accuracy of a few tens of nanometers ${ }^{32}$ by the application of digital image correlation (DIC). ${ }^{33,34}$ It is, thus, not a prerequisite to use electron microscopy to obtain accurate engineering stress-strain curves from nanomechanical experiments.

This last point was implemented in this article along with a MEMS-based platform to investigate the mechanical behavior of polymeric nanofibers subjected to large strains and micronewton forces. The test device, fabricated by surface micromachining, consisted of specimen grips, a leafspring load cell, and a grip for actuation by an external piezoelectric transducer (PZT). The load cell stiffness was fine tuned with the aid of focused ion beam (FIB) to increase the accuracy in force measurement from polymeric nanofibers with a range of diameters. The fiber elongation and tensile force were obtained simultaneously and independently from optical measurements processed by an in-house developed DIC computer program.

\section{EXPERIMENTAL METHOD}

The experimental apparatus employed a surface micromachined platform for nanofiber gripping and measurement of the force in the fiber. The latter was performed by an integrated leaf-spring load cell. One end of the nanofiber was attached to the leaf-spring supported grip. The other end was held fixed (stationary grip) during fiber drawing. For this purpose, a tipless AFM cantilever attached to a three-axis stage was mounted on the stationary grip (Fig. 1) using an epoxy adhesive. Each die, hosting approximately 100 MEMS platforms similar to that in Fig. 1 was mounted on a linear piezoelectric (PZT) stage. 
The MEMS platform was fabricated using conventional surface micromachining with single mask process. The fabrication started with the growth of a $2.0 \mu \mathrm{m}$ thick silicon dioxide on a (100) silicon wafer, followed by a deposition of $2.0 \mu \mathrm{m}$ of silicon dioxide through low pressure chemical vapor deposition (LPCVD) using silane and oxygen gases, to create a $4.0 \mu \mathrm{m}$ thick silicon dioxide insulating and sacrificial layer. Polysilicon with a thickness of $5.2 \mu \mathrm{m}$ was then deposited using LPCVD and silane and annealed at $1050^{\circ} \mathrm{C}$ to from a fine-grained polycrystalline microstructure. Next, a $0.3 \mu \mathrm{m}$ silicon dioxide layer was deposited using LPCVD to serve as a mask to pattern the polysilicon. The masking silicon dioxide was then patterned using standard photolithography techniques, followed by plasma dry etching, which was followed by plasma dry etching of the polysilicon. Finally the devices were released in hydrofluoroulkane (HFA) which etched away the undesired masking and sacrificial silicon dioxide. Further details about the fabrication can be found in Ref. 28.

The test samples were electrospun polyacrylonitrile (PAN) nanofibers fabricated from solution of PAN in dimethylformamide, ${ }^{35}$ with average molecular weight of 150 000 . The diameters of the fibers were in the range of $300-600 \mathrm{~nm}$ while two fiber lengths of 25 and $50 \mu \mathrm{m}$ were selected.

The experimental apparatus was designed to allow for experiments inside an SEM. However, physical aging and cross-linking in polymeric fibers subjected to the SEM electron beam do not permit its use. ${ }^{31}$ Instead, the tension tests were performed under an optical microscope at 500X magnification by recording digital images with 1280 $\times 1024$ pixel resolution. The nanofiber diameter was prohibitively small $(300-600 \mathrm{~nm})$ to view the fiber and measure its elongation directly by using optics. Thus, optical images of the test platform were used to measure the cross-head displacements by resolving displacements of the fiber grips and the deflection of the center of the doubly supported beam comprising the load cell. Images of the test platform contained both force and displacement records and thus force and stretch ratio data were acquired synchronously. The sample grips were subject to rigid body motions that can be resolved with resolution significantly better than the pixel size by using DIC. ${ }^{32}$ Specifically, the rigid body motion of each grip was computed as the average of the motion of an area of $100 \times 100$ pixels.

It is generally accepted that the accuracy of rigid body motion calculations by DIC is at least as good as 1/8 of a data pixel $^{34}$ or better. ${ }^{38}$ The displacement resolution verified in this work was $1 / 8$ of a pixel, which is translated into $50 \mathrm{~nm}$ or better. The benefit of this method is that strain rate experiments can be conducted and the limiting rate of loading is defined by the frame rate of the charge coupled device (CCD) camera. DIC calculations require a sufficiently dense speckle pattern. The $5.2 \mu \mathrm{m}$ polysilicon had a rough surface that scattered light to generate a natural speckle pattern for the application of DIC.

The nanofibers were isolated from a deposition grid and cut in sections of $200-300 \mu \mathrm{m}$ long by local melting by using a scanning thermal microscopy probe. Specimens were

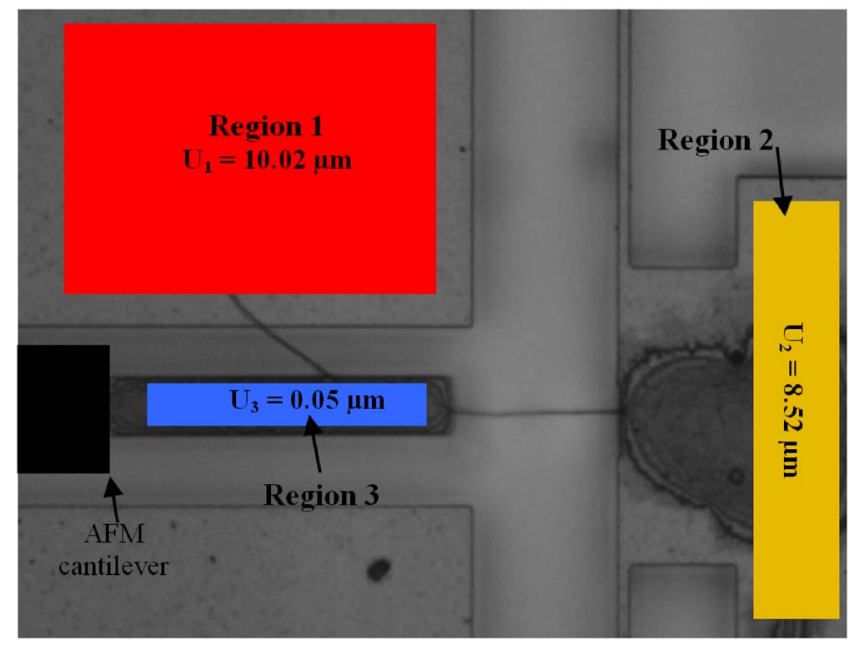

FIG. 2. DIC contour plots of rigid body displacements monitored during tension. Each region has a solid color due to its rigid body motion. The fiber length in this figure is $25 \mu \mathrm{m}$.

substantially larger than the 25 or $50 \mu \mathrm{m}$ space between the grips to allow for adhesive gripping and for a free fiber segment that served as reference for postmortem SEM imaging and determination of the undeformed diameter. This approach eliminated the need for exposure to an SEM before testing. Tests showed that the exposure of polymer nanofibers to an SEM caused significant reduction in ductility by as much as $90 \%$.

After specimen mounting, a tipless AFM cantilever was attached to the stationary grip (Fig. 1) using an epoxy glue with the aid of a linear translation stage. The tethers supporting the stationary grip are not necessary after mounting the AFM probe. They guarantee the application of in-plane force on the fiber keeping the MEMS platform in focus during optical imaging. However, they provided a constraint when long polymeric fibers were subjected to large stretch ratios. For this reason, they were broken before testing with the aid of a probe station. During testing, the AFM cantilever remained stationary while the PZT translated the chip carrying the MEMS platform, thus stretching the nanofiber.

Tension tests under monotonic and cyclic loading were performed. The former is suitable to measure the fiber strength and strain at failure, while cyclic loading was employed to measure the elastic modulus of the fibers from the unloading curves. To extract force-elongation curves, images of the MEMS device recorded during testing were compared with its unloaded configuration by DIC. The rigid body motions of three parts of the device were monitored: $U_{1}$ at the substrate (region 1 in Fig. 2), $U_{2}$ at the freestanding load sensor (region 2 in Fig. 2), and $U_{3}$ at the grip where the AFM cantilever is attached (region 3 in Fig. 2), which served as reference. The applied force on the fiber was then calculated as the load cell stiffness times the deflection of the load cell, which was equal to $u_{l}=U_{2}-U_{1}$. The motion of the freestanding part of the load cell relative to the AFM cantilever grip was used to calculate the elongation of the nanofiber, $u_{f}$ $=U_{2}-U_{3}$. A snapshot of computed contours of rigid body displacements is shown in Fig. 2.

The undeformed fiber diameter and length were mea- 


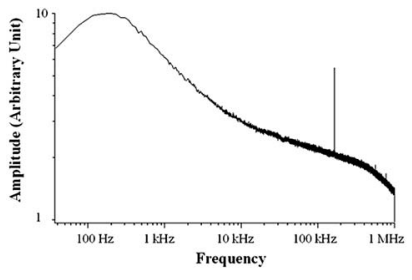

(a)

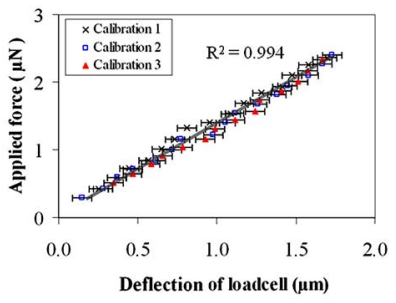

(b)
FIG. 3. (a) Frequency spectrums of AFM cantilever. (b) Calibration curves of the load cell. Three independent calibrations of the same load cell are shown.

sured by SEM and optical imaging, respectively. These quantities were used to extract the engineering stress $\sigma=k_{l} u_{l} / A_{0}$ and strain $\varepsilon=u_{f} / l_{0}$ in the fibers from the force-elongation curves, where $k_{l}$ was the load cell stiffness, $l_{0}$ was the initial length of the fiber, and $A_{0}$ was the initial cross sectional area of the fiber.

The device load cell was calibrated by an AFM cantilever of known stiffness $\left(k_{c}\right)$, that was determined by analyzing the frequency spectrum of its thermal fluctuations, ${ }^{36}$ as shown in Fig. 3(a). The nominal stiffness of the AFM cantilever was chosen to be close to the calculated stiffness of the load cell in order to minimize the uncertainty in the load cell calibration. ${ }^{37}$ The AFM cantilever was held vertically and pressed against the load cell, and optical images of the load cell were used to determine the deformation of the load cell $\left(u_{l}\right)$ and the AFM cantilever $\left(u_{c}\right)$ by using DIC. Since the load cell and the AFM cantilever were in equilibrium, $k_{l} u_{l}$ $=k_{c} u_{c}$ and $u_{c}=U_{2}$, because the base of the cantilever was held fixed. A load cell calibration curve is shown in Fig. 3(b). The stiffness of the particular load cell was $1.33 \pm 0.03 \mathrm{~N} / \mathrm{m}$, which is close to $1.38 \mathrm{~N} / \mathrm{m}$ calculated from a finite element analysis. The difference between the two values comes from the uncertainty in the dimensions of the beam and the rigidity of the supports (boundary conditions) of the load cell. This uncertainty can be significantly larger, and, thus, the experimental measurement of the $k_{l}$ is recommended. ${ }^{36}$

The stiffness of the load cells used in our experiments was chosen based on calibration experiments with actual fibers that provided the fiber yield stress. Special care was taken so that the load cell deflections were sufficiently large to provide high force resolution in the elastic deformation regime, with the load cell motion still remaining planar. For the tests performed, the length of load cell beams was tuned to produce $1.3 \mathrm{~N} / \mathrm{m}$ stiffness, which corresponds to a $6 \mu \mathrm{m}$ load cell deflection at fiber yield. In order to perform tests with thinner fibers, the length of the load cell tethers can be increased by depositing the Pt blocks closer to the load cell anchor points. The maximum length of the tether length in our current design is $400 \mu \mathrm{m}$, resulting in a load cell stiffness of $0.11 \mathrm{~N} / \mathrm{m}$. Assuming a minimum of ten data points acquired before yield at $60 \mathrm{MPa}$ to capture the linear response of a polymer nanofiber, and considering the fact the minimum resolved load cell deflection is $65 \mathrm{~nm}$, which is the DIC resolution measured in the following section, the estimated diameter of the thinnest fiber to be tested with this setup is $40 \mathrm{~nm}$.

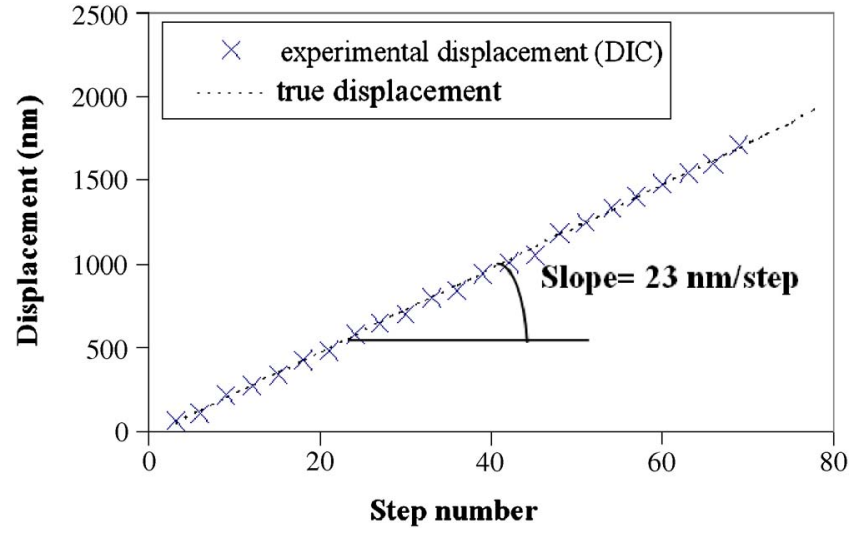

FIG. 4. Rigid body displacement of PZT actuator as determined by DIC $(\times)$ as a function of actuator step. The dashed line (true displacement) is the position of the actuator for a step size of $23 \mathrm{~nm}$.

\section{A. Uncertainty analysis}

DIC has been shown to resolve displacements on the order of a few tens of nanometers using optical imaging. ${ }^{32,38}$ The displacement accuracy depends on the imaging conditions, the signal-to-noise ratio of the imaging system, and the displacement distribution (gradient). Large correlation squares in DIC application favored better accuracy in capturing rigid body motions. In an effort to assess the limits of DIC, the PZT was actuated at constant speed with a nominal step size of $23 \mathrm{~nm}$, and the rigid body motion of the MEMS chip was recorded optically. The rigid body displacement at each step was calculated by using DIC and plotted as a function of the PZT step order. The true displacement of the actuator was then calculated from the step number and the nominal step size (considered constant), and it is represented by the straight line in Fig. 4. The true displacement of the actuator matched very well the actuator position as calculated by DIC, which in Fig. 4 is indicated as the experimental displacement. The maximum difference between two sets of data was approximately $45 \mathrm{~nm}$. Therefore, by virtue of propagation of error, the uncertainty in measuring the relative displacement of two regions on the test platform (i.e., regions 1 and 2 in Fig. 2$)$ is at most $45 \sqrt{2}(=\sim 65) \mathrm{nm}$. This uncertainty includes the noise in the optical images and the uncertainty in capturing an image frame at a given, nonzero, exposure time during actuator motion. The $65 \mathrm{~nm}$ uncertainty is approximately $1 / 8$ of the average wavelength of light used in the experiments which correlates well with previous reports about the resolution of DIC. ${ }^{32}$ Since the uncertainty is not cumulative, measurement of large rigid body motions minimizes its relative contribution to the overall displacement. Thus, longer fibers improve the accuracy in determining stress-strain curves.

Given this measurement accuracy, we can compute the relative uncertainty in the calculated quantities. The uncertainty in computing the engineering strain $\left(\varepsilon=u_{f} / l_{0}\right)$ in the nanofiber is 


$$
\begin{aligned}
(\Delta \varepsilon)^{2} & =\left(\frac{d \varepsilon}{d u}\right)^{2}(\Delta u)^{2}+\left(\frac{d \varepsilon}{d l_{0}}\right)^{2}\left(\Delta l_{0}\right)^{2} \\
& =\left(\frac{\Delta u}{l_{0}}\right)^{2}+\varepsilon^{2}\left(\frac{\Delta l_{0}}{l_{0}}\right)^{2},
\end{aligned}
$$

where $\Delta()$ is the uncertainty in the measurement of each independent variable. Therefore, the engineering strain in a sample with initial gauge length of $25 \pm 0.5 \mu \mathrm{m}$ is bounded to $0.26 \%$, and it substantially improves as the sample length increases. Also, for engineering stress $\left(\sigma=k_{l} u_{l} / A_{0}\right)$,

$$
\begin{aligned}
(\Delta \sigma)^{2} & =\left(\frac{d \sigma}{d k}\right)^{2}(\Delta k)^{2}+\left(\frac{d \sigma}{d u_{l}}\right)^{2}\left(\Delta u_{l}\right)^{2}+\left(\frac{d \sigma}{d A_{0}}\right)^{2}\left(\Delta A_{0}\right)^{2} \\
& =\sigma^{2}\left(\frac{\Delta k}{k}\right)^{2}+\left(\frac{k}{A_{0}}\right)^{2}\left(\Delta u_{l}\right)^{2}+\sigma^{2}\left(\frac{\Delta A_{0}}{A_{0}}\right)^{2}
\end{aligned}
$$

Finally, the uncertainty in computing the stiffness of the load cell $\left(k_{l} u_{l}=k_{c} u_{c}\right)$ is

$$
\begin{aligned}
\left(\Delta k_{l}\right)^{2} & =\left(\frac{\partial k_{l}}{\partial k_{c}}\right)^{2}\left(\Delta k_{c}\right)^{2}+\left(\frac{\partial k_{l}}{\partial u_{l}}\right)^{2}\left(\Delta u_{l}\right)^{2}+\left(\frac{\partial k_{l}}{\partial u_{c}}\right)^{2}\left(\Delta u_{c}\right)^{2} \\
& =\left(k_{l}\right)^{2}\left(\frac{\Delta k_{c}}{k_{c}}\right)^{2}+\left(\frac{k_{c} u_{c}}{u_{l}^{2}}\right)^{2}\left(\Delta u_{l}\right)^{2}+\left(\frac{k_{c}}{u_{l}}\right)^{2}\left(\Delta u_{c}\right)^{2} .
\end{aligned}
$$

The method applied to measure the stiffness of the AFM cantilever is considered to be among the most accurate, and the uncertainty $\Delta k_{c} / k_{c}$ has been reported to be as good as $5 \%{ }^{39}$ The repeatability of three measurements of the cantilever stiffness was very good with the standard deviation of $0.03 \mathrm{~N} / \mathrm{m}$.

This relative uncertainty in engineering stress can be further reduced by improving the accuracy in the undeformed fiber cross section and by employing a compliant load cell. For an initial fiber diameter of $400 \pm 10 \mathrm{~nm}$ and engineering stresses below $40 \mathrm{MPa}$, the uncertainty in engineering stress for a typical load cell with $1.5 \pm 0.15 \mathrm{~N} / \mathrm{m}$ stiffness is about 4.6 MPa, which can be further reduced by improving the accuracy in the stiffness of the AFM cantilever used to calibrate the load cell.

Furthermore, if the uncertainty in the initial length and diameter of the nanofibers and the stiffness of the loadcell are negligible, such that only the uncertainties in $U_{1}, U_{2}$, and $U_{3}$ need to be considered for, Eqs. (1) and (2) are reduced to $\Delta \varepsilon=\Delta u / l_{0}$ and $\Delta \sigma=k / A_{0} \Delta u_{l}$, respectively. In this case, for a fiber with an initial length of $25 \mu \mathrm{m}$, initial diameter of $400 \mathrm{~nm}$, and for a load cell with the stiffness of $1.5 \mathrm{~N} / \mathrm{m}$, the uncertainties in engineering strain and stress are $\sim 0.25 \%$ and $0.78 \mathrm{MPa}$, respectively.

\section{RESULTS AND DISCUSSION}

The MEMS platform was used to subject nine PAN nanofibers to monotonic loading and four PAN nanofibers to cyclic loading. Loadings and unloadings were performed at the nominal strain rate of $2.5 \times 10^{3} \mathrm{~s}^{-1}$, and the forceelongation curves were determined according to the discussion in Sec. II. Subsequent to each tension test, the undeformed segment of the fibers, not included in the gage

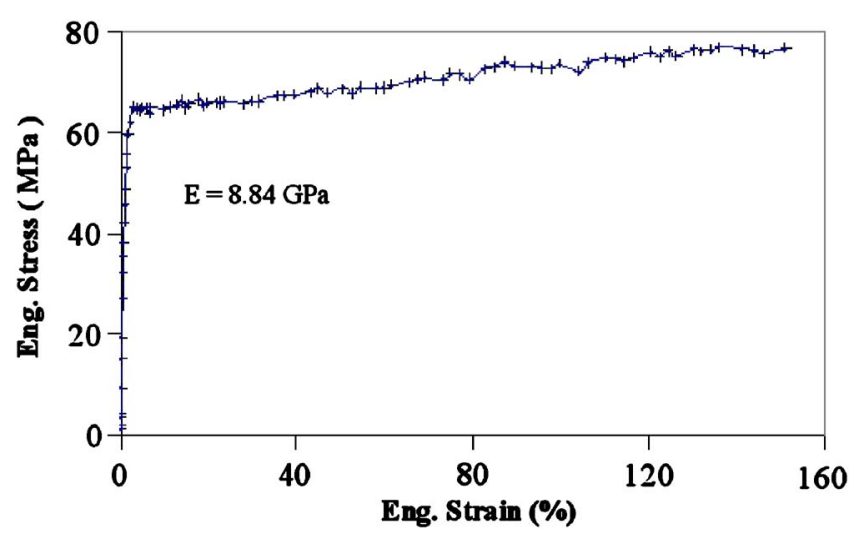

FIG. 5. Engineering strain-stress curve of an electrospun PAN nanofiber loaded at $2.5 \times 10^{3} \mathrm{~s}^{-1}$.

section, was imaged with a SEM to measure the initial fiber diameter and calculate the engineering stress as the ratio of the force on the fiber to the initial cross section area. The fibers were sputter coated with $10 \mathrm{~nm}$ of gold for improved resolution. The coating also protected the fibers against the shrinkage that could happen due to e-beam. For most samples, the standard deviation in measuring the initial fiber diameter at different locations along the fiber was less than $20 \mathrm{~nm}$.

An engineering stress-strain curve under monotonic loading is shown in Fig. 5. The fibers behave elastically until $5 \%-10 \%$ engineering strain followed by large elongation and no softening. The nanofibers did not deform in a homogeneous manner as implied by the SEM images of undeformed and deformed nanofibers in Figs. 6(a) and 6(b). The ductility varied between $150 \%$ and $220 \%$ for different diameters and gauge lengths, being several times larger than the ductility of PAN precursor microfibers fabricated by drawing ${ }^{40}$ (which does not exceed $30 \%$ ) and dry-jet-wet spinning. ${ }^{41}$ This may be attributed in part to the enhanced mobility of surface macromolecules that are less constrained compared to bulk macromolecules, and the higher ratio of surface-to-interior polymer molecules in thinner samples, which increased the contribution of surface molecules to the overall mechanical response. In polymeric nanofibers, the tensile load is transferred through the entanglement network of chains with significantly smaller constrains from lateral entanglements compared to bulk. ${ }^{42}$ On the other hand, in polymeric microfibers and bulk polymers, lateral entanglements prohibit plastic
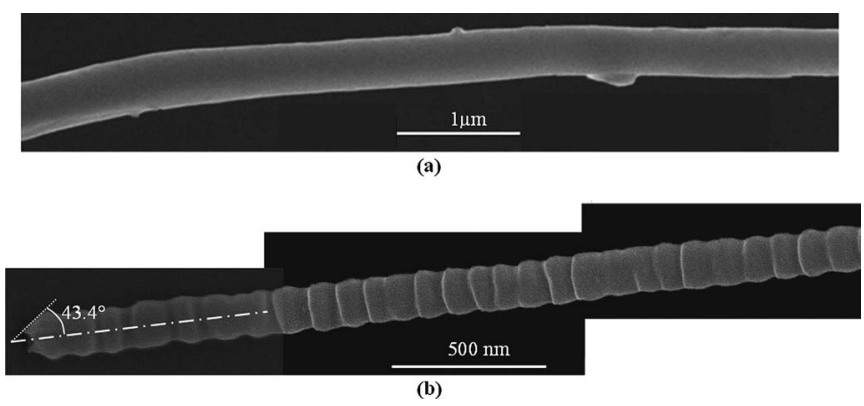

FIG. 6. SEM images of (a) undeformed PAN nanofiber and (b) deformed PAN nanofiber, with multiple surface ripples formed during drawing. The fracture surface is typical for ductile fracture. 

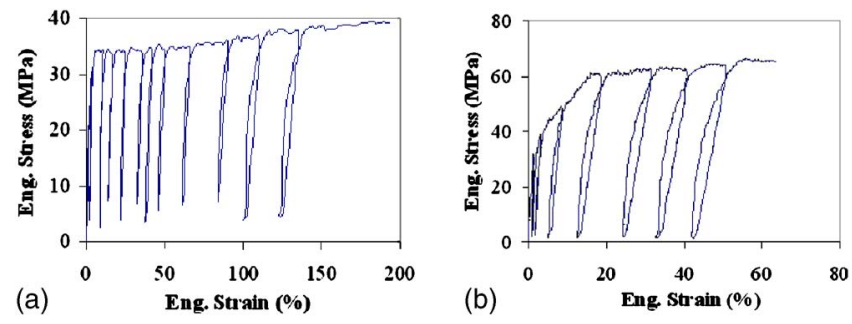

FIG. 7. Engineering stress-strain curves from two electrospun PAN nanofibers. (a) Sharp rise in stress is accompanied by plastic flow with small effective stiffening. (b) Reduced fiber ductility and increased strength accompanied by nonlinear stress-strain curve before saturation stress.

flow by developing hydrostatic pressure. As a result, cavities form during loading, leading to crack formation and premature failure. This reduction in lateral constraints may also lead to smaller fiber strength compared to bulk.

The engineering fiber strength varied between 30 and $71 \mathrm{MPa}$ depending on fiber diameter. The elastic modulus, measured from unloading curves at strains less than $5 \%$ was 7.6 $\pm 1.5 \mathrm{GPa}$, where $1.5 \mathrm{GPa}$ was the standard deviation. This tensile strength and modulus of elasticity were in general agreement with the work by Fennessey and Farris who tested twisted yarns of PAN. ${ }^{43}$ They reported the strength and modulus to be in the range of $70-160 \mathrm{MPa}$ and 4-7 GPa, respectively. On the other hand, the elastic modulus is about a third of the value reported by Yua et al. for PAN electrospun nanofibers with diameters of $200 \mathrm{~nm},{ }^{18}$ and it is much smaller than the values obtained by Chae et al. for dry-jetwet spun PAN microfibers. ${ }^{41}$

A comparison between deformed and undeformed PAN nanofibers, Figs. 6(a) and 6(b), shows multiple necks forming on the fibers during testing and shear failure at ultimate stress. The fracture surface formed an approximate angle of $45^{\circ}$ with the fiber axis at the locus of the maximum shear stress. The formation of multiple necks with consistent amplitude and periodicity was prominent in all fibers. Each neck was the result of a local instability that resulted in increase of the local stress. Large axial stresses generate significant shear stress that upon neck initiation allows for macromolecular rotation at the neck. Except for minor stiffening, the formation of multiple necks, Fig. 6(b), without neck propagation did not allow for a rise in the engineering stressstrain curve, as seen in Fig. 5. This process is equivalent to cold drawing, but the induced cross-head displacement to the nanofiber is localized at each neck without substantial increase in the applied force. The average neck wavelength for fibers with different diameters, in Fig. 6(b), was $150-200 \mathrm{~nm}$, and in general independent of the fiber diameter. In addition, the neck amplitude, computed as half of the difference between the fiber average diameter and the fiber diameter at the neck, was $60-100 \mathrm{~nm}$ for fibers with undeformed diameters of $300-500 \mathrm{~nm}$. The process of seeding the numerous necks seen in Fig. 6(b) was strain rate dependent and it is the subject of a follow up article.

The modulus of elasticity cannot be determined reliably from the loading curves such as that in Fig. 5. Instead, loading-unloading stress-strain curves were recorded, according to Figs. 7(a) and 7(b) and the elastic modulus was

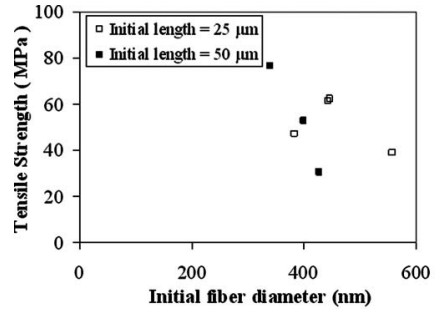

(a) (b)

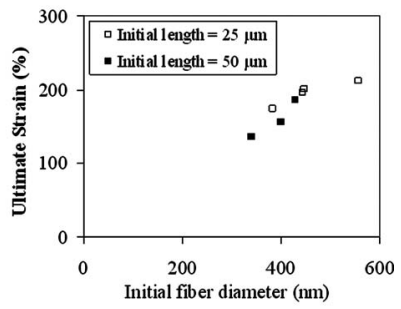

FIG. 8. (a) Dependence of engineering tensile strength and (b) ultimate strain on undeformed nanofiber diameter.

calculated from the unloading paths at strains less than $5 \%$. Within the measurement accuracy and at fiber strains as large as $10 \%$, the unloading curves were linear and of the same slope as the loading curves. At larger strains, each unloading curve began with a sharp stress relaxation, while the fiber length remained constant. Further unloading was accompanied by smoother reduction in engineering strain, which indicates the dependence of stress relaxation rate on the magnitude of the applied stress. The hysteresis in loadingunloading curves at large strains has been attributed to stress relaxation in the fiber due to the large stress in the beginning of unloading. ${ }^{44}$

Two types of engineering stress-strain curves were observed and are shown in Figs. 7(a) and 7(b). In the first, a sharp rise of stress under constant slope occurred before yielding with subsequent constant stress during fiber drawing. In the second plot, Fig. 7(b), fiber softening took place until $\sim 20 \%$ strain, when the force in the fiber reached a plateau that was significantly larger than that in Fig. 7(a). These two types of behavior are reminiscent of the mechanical response of pristine and predrawn polycarbonate samples by cold drawing as described by Zhou et $a l^{44}$ and Masud and Chudnovsky. ${ }^{45}$ The nanofibers that was used to determine the mechanical properties of PAN in this work are those following the behavior in Fig. 7(a), because they were considered pristine fibers, as opposed to fibers that followed the $\sigma$ - $\varepsilon$ curve in Fig. 7(b) that were considered to have been subjected to predrawing with preexisting necks or were characterized by large fluctuations in diameter.

In terms of dimensional scaling, the two geometry parameters, fiber diameter and length, did have an effect on the fiber mechanics. Smaller fiber diameters correlated with increased fiber strength. This precipitous trend may be explained by enhanced molecular alignment occurring during electrospinning as thinner fibers are expected to be the result of larger drawing ratios. ${ }^{12}$ The opposite trend, in a more definitive fashion, was observed for the ultimate strain, Fig. 8(b). Fibers of larger diameters failed at larger stretch ratios. The reduced ductility for thinner nanofibers further supports the argument that they were subjected to molecular alignment during fabrication, in accordance with the conclusion derived from the strength data in Fig. 8(a). Fiber ductility decreased slightly with fiber length potentially due to statistically larger number of defects in longer fibers. 


\section{CONCLUSIONS}

A novel method for the investigation of the mechanical behavior of nanofibers and nanowires with special emphasis on highly deformable polymeric nanofibers was presented. The method was used to measure the mechanical response of electrospun PAN nanofibers with diameters between 300 and $600 \mathrm{~nm}$ at a nominal strain rate of $2.5 \times 10^{-3} \mathrm{~s}^{-1}$ and at room temperature. The strain and force in the sample were calculated from optical images and by virtue of DIC. The modulus of elasticity of the electrospun PAN nanofibers as recorded from unloading curves was $7.6 \pm 1.5 \mathrm{GPa}$, and it was equal to that reported before for yarns from twisted PAN electrospun nanofibers ${ }^{43}$ without significant molecular alignment. The lack of significant alignment was further corroborated by the large ultimate strain recorded for all samples. Thinner nanofibers supported higher strengths and reduced ductility compared to thicker fibers, which provides indirect evidence that thinner fibers are characterized by enhanced molecular alignment induced during electrospinning. For most of the electrospun PAN nanofibers, the engineering stress-strain curves were elastic-nearly perfectly plastic with ductilities reaching $220 \%$ in the thicker fibers. These large fiber ductilities may also be supported by the reduced lateral entanglement of nanofiber surface macromolecules, which prevented the development of hydrostatic stresses, subsequent formation of cavities, and premature failure, which are common in bulk.

The advantage of the method presented here is that the load and deformation in the fiber are concurrently measured without the need for synchronization of data recording. Thus, fibers can be tested at strain rates varying by many orders of magnitude and at different temperatures. The application of optical instead of electron microscopy also addresses a significant limitation: the vulnerability of polymeric nanofibers to electron beam radiation.

\section{ACKNOWLEDGMENTS}

The authors acknowledge the financial support by the National Science Foundation (NSF) under NSF-NIRT Grant No. DMI-0403876 (DMI-0532320) with Dr. J. Chen and Dr. K. Lyons as the program managers, and by the U.S. Army Research Office under Grant No. W911NF-06-1-0356 with Dr. Bruce LaMattina as the program manager. The authors also thank Scott MacLaren for his help in determining the spring constant of the AFM cantilevers and Vania Petrova for her helps in imaging the nanofibers using SEM.

${ }^{1}$ S. Sahoo, H. W. Ouyang, J. C. H. Goh, T. E. Tay, and S. L. Toh, Proc. SPIE 5852, 658 (2005).

${ }^{2}$ W. L. Li, C. T. Laurencin, E. J. Caterson, R. S. Tuan, and F. K. Ko, J. Biomed. Mater. Res. 60, 613 (2002).

${ }^{3}$ R. S. Barhate, C. K. Loong, and S. Ramakrishna, J. Membr. Sci. 283, 209 (2006).

${ }^{4}$ M. M. Bergshoef and G. J. Vancso, Adv. Mater. (Weinheim, Ger.) 11, 1362 (1999).
${ }^{5}$ T. Ondarcuhu and C. Joachim, Europhys. Lett. 42, 215 (1998).

${ }^{6}$ B. B. Lakshmi, C. J. Patrissi, and C. R. Martin, Chem. Mater. 9, 2544 (1997).

${ }^{7}$ Z. M. Huang, Y. Z. Zhang, M. Kotaki, and S. Ramakrishna, Compos. Sci. Technol. 63, 2223 (2003).

${ }^{8}$ B. D. Li, Y. Wang, and Y. Xia, Adv. Mater. (Weinheim, Ger.) 16, 361 (2004).

${ }^{9}$ E. Zussman, D. Rittel, and A. L. Yarin, Appl. Phys. Lett. 82, 3958 (2003).

${ }^{10}$ E. Zussman, A. Theron, and A. L. Yarin, Appl. Phys. Lett. 82, 973 (2002).

${ }^{11}$ F. Ko, Y. Gogotsi, A. Ashraf, N. Naguib, H. Ye, G. Yang, C. Li, and P. Willis, Adv. Mater. (Weinheim, Ger.) 15, 1161 (2003).

${ }^{12}$ E. P. S. Tan and C. T. Lim, Compos. Sci. Technol. 66, 1102 (2006).

${ }^{13}$ S. Cuenot, S. Demoustier-Champagne, and B. Nysten, Phys. Rev. Lett. 85, $1690(2000)$

${ }^{14}$ S. Y. Gu, Q. L. Wu, J. Ren, and G. J. Vancso, Macromol. Rapid Commun. 26, 716 (2005).

${ }^{15}$ B. Wu, A. Heidelberg, and J. Boland, Nat. Mater. 4, 525 (2005).

${ }^{16}$ E. Zussman, X. Chen, W. Ding, L. Calabri, D. A. Dikin, J. P. Quintana, and R. S. Ruoff, Carbon 43, 2175 (2005).

${ }^{17}$ M. F. Yu, M. S. Atashbar, and X. Chen, IEEE Sens. J. 5, 20 (2005).

${ }^{18}$ P. A. Yua, Y. Wen, J. A. Turner, Y. A. Dzenis, and Z. Li, Appl. Phys. Lett. 90, 111909 (2007).

${ }^{19}$ M. A. Haque and M. T. A. Saif, Exp. Mech. 43, 248 (2003).

${ }^{20}$ E. Zussman, M. Burnman, A. L. Yarin, R. Khalfin, and Y. Cohen, J. Polym. Sci., Part B: Polym. Phys. 44, 1482 (2006).

${ }^{21}$ E. P. S. Tan, C. N. Goh, C. H. Sow, and C. T. Lim, Appl. Phys. Lett. 86, 073115 (2005)

${ }^{22}$ M. F. Yu, B. S. Files, S. Arepalli, and R. S. Ruoff, Phys. Rev. Lett. 84, 5552 (2003).

${ }^{23}$ E. P. S. Tan and C. T. Lim, Rev. Sci. Instrum. 75, 2581 (2004).

${ }^{24}$ R. Inai, M. Kotaki, and S. Ramakrishna, Nanotechnology 16, 208 (2005).

${ }^{25}$ S. Lu, Z. Guo, W. Ding, and R. S. Ruoff, Rev. Sci. Instrum. 77, 056103 (2006).

${ }^{26}$ Y. Zhu, N. Moldovan, and H. D. Espinosa, Appl. Phys. Lett. 86, 013506 (2005).

${ }^{27}$ M. Kiuchi, Y. Isono, S. Sugiyama, T. Morita, and S. Matsui, Proceedings of the Fifth IEEE Conference on Nanotechnology, Nagoya, Japan, 2005, Vol. 2, p. 673.

${ }^{28}$ H. Kahn, R. Ballarini, R. L. Mullen, and H. Heuer, Proc. R. Soc. London, Ser. A 455, 3807 (1999).

${ }^{29}$ V. P. Jaecklin, C. Linder, N. F. De Rooij, J. M. Moret, R. Bischof, and F. Rudolf, J. Microelectromech. Syst. 92, 147 (1992).

${ }^{30}$ B. A. Samuel, M. A. Haque, B. Yi, R. Rajagopalan, and H. C. Foley, Nanotechnology 18, 1 (2007).

${ }^{31}$ L. Reimer and A. Schmidt, Scanning 7, 47 (1985).

${ }^{32}$ T. A. Berfield, J. K. Patel, R. G. Shimmin, P. V. Braun, J. Lambros, and N. R. Sottos, Small 2, 631 (2006).

${ }^{33}$ T. Chu, W. H. Peters, W. F. Ranson, and M. A. Sutton, Proc. Soc. Exp. Stress Anal. 39, 73 (1982).

${ }^{34}$ G. Vendroux and W. G. Knauss, Exp. Mech. 38, 86 (1998).

${ }^{35}$ Y. Dzenis and Y. Wen, Mater. Res. Soc. Symp. Proc. 702, 173 (2002).

${ }^{36}$ J. L. Hutter and J. Bechhoefer, Rev. Sci. Instrum. 64, 1868 (1993).

${ }^{37}$ A. Torii, M. Sasaki, K. Hane, and S. Okuma, Meas. Sci. Technol. 7, 179 (1996).

${ }^{38}$ B. Serio, J. J. Hunsinger, and B. Cretin, Rev. Sci. Instrum. 75, 3335 (2004).

${ }^{39}$ N. A. Burnham, X. Chen, C. S. Hodges, G. A. Matei, E. J. Thoreson, C. J. Roberts, M. C. Davies, and S. J. B. Tendler, Nanotechnology 14, 1 (2003).

${ }^{40}$ Q. Xu, L. Xu, W. Cao, and S. Wu, Polym. Adv. Technol. 16, 642 (2005).

${ }^{41}$ H. G. Chae, T. V. Sreekumar, T. Uchida, and S. Kumar, Polymer 46, 10925 (2005).

${ }^{42}$ G. H. Michler, H. H. Kausch, and R. Adhikari, J. Macromol. Sci., Phys. B45, 727 (2006).

${ }^{43}$ S. F. Fennessey and R. J. Farris, Polymer 45, 4217 (2004).

${ }^{44}$ Z. Zhou, A. Chudnovsky, C. P. Bosnyak, and K. Sehanboish, Polym. Eng. Sci. 35, 304 (1995).

${ }^{45}$ A. Masud and A. Chudnovsky, Int. J. Plast. 15, 1139 (1999). 